\title{
Efficacy and safety of neoadjuvant chemotherapy and immunotherapy in locally resectable advanced esophageal squamous cell carcinoma
}

\author{
Zhigang Wu ${ }^{1,2 \#}$, Qiang Zheng ${ }^{2,3 \#}$, Haiquan Chen ${ }^{1,2}$, Jiaqing Xiang ${ }^{1,2}$, Hong Hu ${ }^{1,2}$, Hang Li ${ }^{1,2}$, \\ Yunjian Pan ${ }^{1,2}$, Yizhou Peng ${ }^{1,2}$, Xingxin Yao ${ }^{1,2}$, Pengcheng Liu ${ }^{1,2}$, Yihua Sun ${ }^{1,2 *}$, Bin Li ${ }^{1,2 *}$, Yawei Zhang ${ }^{1,2 *}$ \\ ${ }^{1}$ Department of Thoracic Surgery, Fudan University Shanghai Cancer Center, Shanghai, China; ${ }^{2}$ Department of Oncology, Shanghai Medical \\ College, Fudan University, Shanghai, China; ${ }^{3}$ Department of Pathology, Fudan University Shanghai Cancer Center, Shanghai, China \\ Contributions: (I) Conception and design: Z Wu, Q Zheng, H Chen, Y Sun, B Li, Y Zhang; (II) Administrative support: H Chen, Y Sun, B Li, Y \\ Zhang; (III) Provision of study materials or patients: Z Wu, Q Zheng, B Li, J Xiang, H Hu, H Li, Y Pan, Y Peng, X Yao, P Liu; (IV) Collection and \\ assembly of data: Z Wu, Q Zheng, Y Pan, Y Peng, X Yao, P Liu; (V) Data analysis and interpretation: Z Wu, H Chen, B Li, J Xiang, H Hu, H Li; (VI) \\ Manuscript writing: All authors; (VII) Final approval of manuscript: All authors. \\ "These authors contributed equally to this work and should be considered as co-first authors. \\ *These authors contributed equally to this work and should be considered as co-corresponding authors. \\ Correspondence to: Yihua Sun; Bin Li; Yawei Zhang. Department of Thoracic Surgery, Fudan University Shanghai Cancer Center, 270 Dong-An Road, \\ Shanghai 200032, China. Email: sun_yihua76@hotmail.com; 1b0256327@hotmail.com; zhangyawei68@hotmail.com.
}

Background: Esophageal squamous cell cancer (ESCC) patients with the potentially resectable disease most would experience relapse after surgery. Immunotherapy has been reported to improve the prognosis of advanced esophageal cancer and may be a new strategy to prevent this urgent condition's recurrence. We first evaluated the efficacy and safety of neoadjuvant chemotherapy combined with immunotherapy in patients with resectable ESCC.

Methods: All patients with resectable locally advanced ESCC (clinical stage III-IVB). Received at least 1 cycle of neoadjuvant chemotherapy combined with immunotherapy (NACI), and the interval between each cycle and the operation should be at least 3 weeks. All patients were treated with standard surgery. The tumor imaginations were obtained at baseline and within a week before surgery. The efficacy endpoint was the rate of major pathologic response (MPR, 10\% viable tumor cells). Expression of immunohistochemicalrelated molecules was investigated in surgical samples.

Results: A total of 38 patients with ESCC were included (36 males, median age 61 years), and most of them used Pembrolizumab (55.26\%) and Camrelizumab (31.58\%). We analyzed 19 patients and found that 13 patients $(68.42 \%)$ achieved radiological partial response (PR) by CT images. R0 resection was performed in 35 patients $(92.11 \%)$, and 10 patients $(26.32 \%)$ developed postoperative complications. Through postoperative pathology, we found 13 (34.21\%) patients had complete pathologic response (cPR), and 16 (42.11\%) patients achieved MPR. We also found that none of the factors had a statistically significant impact on MPR. Still, the regression rate of Sum of lesion diameter (SLD) was significantly positively correlated with the pathological remission rate $(\mathrm{P}=0.012, \mathrm{r}=0.565)$.

Conclusions: The rate of MPR in ESCC patients reached $42.11 \%$. The use of the NACI regimen did not increase the occurrence of complications in neoadjuvant treatment and operation, and the SLD regression rate has a certain guiding significance for the effect of immunotherapy.

Keywords: Neoadjuvant immunochemotherapy; immunotherapy; chemotherapy; locally advanced esophageal squamous cell carcinoma

Submitted Feb 28, 2021. Accepted for publication May 02, 2021.

doi: $10.21037 /$ jtd-21-340

View this article at: http://dx.doi.org/10.21037/jtd-21-340

(c) Journal of Thoracic Disease. All rights reserved. 


\section{Introduction}

Esophageal squamous cell cancer (ESCC) is one of the most malignant cancers (1). For locally advanced stages, the five-year survival period is frustrating, ranging from $17-23 \%$, which may be slightly higher in patients with resectable diseases (2-5), and the current National Comprehensive Cancer Network (NCCN) guidelines recommend neoadjuvant therapy (6). Now, immunotherapy as a new treatment is effective when used alone in resectable esophageal cancer (7). More and more evidence of neoadjuvant immunotherapy suggests that it can improve the prognosis of unresectable tumors, and immunotherapy drugs have become diverse, such as "Camrelizumab, Pembrolizumab or Sintilimab," etc. (8), which have all been proven to be effective and safe for treatment.

Previously, the prognosis of surgical treatment of patients with locally advanced disease after neoadjuvant chemotherapy has improved, and the five-year survival rate is between 26-36\% (9). In the KEYNOTE and ATTRACTION studies, immunotherapy significantly improved the five-year survival rate of advanced ESCC $(10,11)$. In this retrospective study, we conducted a survey of the efficacy and safety of surgery after neoadjuvant chemotherapy combined with immunotherapy (NACI) and explored to compare the effect differences between different immunodrugs.

We present the following article in accordance with the STROBE reporting checklist (available at http://dx.doi. org/10.21037/jtd-21-340).

\section{Methods}

\section{Patients}

This study included 38 patients with resectable locally advanced ESCC. The study was conducted in accordance with the Declaration of Helsinki (as revised in 2013). This study was approved by the Shanghai Cancer Center (IRB\# 090977-1). Written informed consent was required from all patients. There was no commercial support for the study. All patients in this study had received more than one course of chemotherapy combined with immunotherapy and were scheduled to undergo radical surgery for esophageal cancer in the Affiliated Cancer Center of Fudan University from November 2019 to December 2020. All tumors met the following criteria: (I) histologically confirmed ESCC, (II) estimated as T [3-4a], N [1-3], and M0, (III) estimated that $\mathrm{R} 0$ resection was accessible, and (IV) the upper margin of the tumor must be at least $3 \mathrm{~cm}$ below the upper esophageal sphincter, and proximal gastric tumors with mild esophageal invasion were excluded. Eligible patients are 18 to 75 years old with Eastern Cooperative Oncology Group (ECOG) $\leq 2$ and lost 10 percent or less of their body weight. Patients must also have adequate hematologic, renal, liver, and lung function without a history of other cancers or previous radiation or chemotherapy.

\section{Staging}

Before and after neoadjuvant therapy, clinical staging (cTNM) was arranged for every patient. It included medical history taking, physical examination, routine laboratory test, echocardiography, pulmonary function test, contrastenhanced chest CT, and upper gastrointestinal endoscopy with biopsy if not previously examined. Ultrasonography of the neck with fine-needle aspiration was performed when cervical lymph node involvement was suspected (9). On CT examinations, thickening of the esophageal wall was diagnosed as T3 and direct involvement of adjacent organs as T4 (12). Some of these patients did not receive CT examination in our institution before neoadjuvant therapy, so two specialized radiologists vaguely classified the stages based on CT reports and film images independently.

\section{Preoperative treatment}

All patients received at least 1 cycle of taxel (docetaxel or paclitaxel) and Platinum Doublet (cisplatin or carboplatin) combined immunotherapy (Camrelizumab, Pembrolizumab, or Sintilimab), and the interval among each cycle and the operation should be at least 3 weeks, such as $0,21,42,63$, etc. Each patient has one and only one triple-drug regimen. The patients were closely monitored for toxic effects of chemotherapy with the use of the National Cancer Institute's Common Terminology Criteria for Adverse Events, version 5.0. For patients with severe side effects, supportive treatment should be given. Techniques for resection of esophageal cancer have been reported (13). All patients were treated with standard minimally invasive esophagectomy (MIE), and the upper tumor mainly was treated with three-incision McKeown surgery (three fields or two fields). The middle and lower segment tumors with two-incision Ivor-Lewis surgery (14). To confirm the maneuverability of the medicine, lung and heart function status was assessed. Besides, the gastric tube was reconstructed with a diameter of 3-4 cm, preserving the right gastroomental artery. End-to-side stapled techniques 
were performed for mechanical cervical anastomoses and all thoracic anastomoses, and the thoracic drainage tube was inserted into the posterior mediastinum.

\section{Postoperative treatment}

Patients are treated roughly the same after surgery if they are physiologically stable. They are extubated at the end of the surgery, admitted to the intensive care unit, and admitted to the thoracic surgery unit the next day. Three days after surgery, the most common method to control postoperative pain is controlled epidural analgesia in patients. On postoperative day (POD) 1, patients were encouraged to prevent blood clots and strengthen enteral nutrition. Patients were given sips of clear liquids on POD 6 and soft solid foods on POD 7, and they were discharged on POD 8 or 9.

\section{Pathological analysis}

Reports on pathological examination described the tumor type, extension, proportion of residual cancer cells, lymph nodes, and resection margins. In the absence of a macroscopic tumor, any abnormal-appearing tissue was paraffin-embedded in total to make an adequate assessment for the presence of a residual tumor and the effects of therapy, which was evaluated under independent microscopes by two pathologists. If a vital tumor was present at $1 \mathrm{~mm}$ or less from the proximal, distal, or circumferential resection margin, it was considered to be microscopically positive (R1). According to Response Evaluation Criteria in Solid Tumors (RECIST) version 1.1 (15), we counted the Sum of lesion diameter (SLD) and Lymph node shortest diameter (LSD) and divided the pathological types after treatment into the following: Complete Response (CR), Partial Response (PR), Progressive Disease (PD), and Stable Disease (SD). We defined major pathological response (MPR) as residual tumor $\leq 10 \%$; Tumor-free cell residue was defined as the complete pathological response (pCR), and the Japanese Classification of Esophageal Cancer was used to evaluate the pathological response (16): grades: Grade 0 (no tumor response), Grade 1a (when necrotic or fibrotic change was observed in less than one third of the tumor), Grade $1 \mathrm{~b}$ (when necrotic or fibrotic change was observed in between one third and two thirds of the tumor), Grade 2 (when more than two thirds of the tumor was necrotic or fibrotic), and Grade 3 (when there were no viable tumor cells). The expression of PD-L1 was defined by the e Combined Positive Score (CPS): (-) CPS $<10$, (+) CPS $\geq 10$.

\section{Statistics}

Demographics and safety, as well as clinical, radiographic, pathologic, and molecular response data, were tabulated using descriptive statistics. Spearman's correlation was used to assess the association. We used the $t$-test, chi-squared test, and Fisher exact test to calculate the correlation of MPR with other variables (age, smoker, drinking history, clinical-stage, etc.), respectively. Cox proportional hazards regression analyses were performed with the following variables: age, smoking history, drinking history, tumor location, interval from completion of NACI to surgery (weeks), immunotherapy regimen, NACI course, Clinical stage. We fit this multivariate Cox regression models to identify the risk factors including all variables. Reported $\mathrm{P}$ values are two-sided, with the significance level set at 0.05. Statistical analyses were performed using software R, version 3.5.1.

\section{Results}

In this study, a total of 38 patients with ESCC were included (36 males, 2 females, median age 61 years, range, 57-75 years), and their median BMI was 22.18 (14.3-29.76). About half of the patients were smokers $(57.89 \%)$ and drinkers (44.74\%), and 25 patients had no basis disease. Among all patients, the middle and lower segment tumors (92.1\%) are the most common. Most of these patients use Pembrolizumab (55.26\%) and Camrelizumab (31.58\%) for immunotherapy and usually undergo esophageal surgery about 5 weeks after treatment (Table 1).

We analyzed CT images of 19 patients before and after NACI. After treatment, we found that 13 patients $(68.42 \%)$ with swollen tumors and lymph nodes shrunk markedly achieved radiological PR, and the remaining 6 patients also had varying degrees of reduction but did not meet the PR criteria. It is worth mentioning that in the course of neoadjuvant therapy, the vast majority of patients were found to have no side effects or slight abnormal white blood cell count. Only one patient developed pulmonary inflammation with a small amount of pleural effusion, and the symptoms disappeared after symptomatic treatment (Table 2).

We mainly performed McKewon (44.74\%) and Ivor Lewis $(55.26 \%)$ surgery on the patients and found that the time of operation $(211.92 \pm 6.57 \mathrm{~min})$ and the amount of blood loss $(186.8 \pm 42.82 \mathrm{~mL})$ after surgery were controlled at a normal level. R0 resection was achieved in 35 patients 
Table 1 Clinical characteristics of patients who underwent chemotherapy and immunotherapy

\begin{tabular}{lc}
\hline Variable & $\mathrm{N}=38(\%)$ \\
\hline Age (medium, range) & $61[57-75]$ \\
Gender & $36(94.74)$ \\
Male & $2(5.26)$ \\
Female & $22.18(14.3-29.76)$ \\
BMl kg/m ${ }^{2}$ (medium, range) & \\
ECOG & $34(89.47)$ \\
1 & $4(10.53)$ \\
2 & \\
Smoking history & $22(57.89)$ \\
Yes & $16(42.11)$ \\
No & \\
Drinking history & $17(44.74)$ \\
Yes & $21(55.26)$ \\
No & \\
\hline
\end{tabular}

Table 1 (continued)
Table 1 (continued)

\begin{tabular}{lc}
\hline Variable & $\mathrm{N}=38(\%)$ \\
\hline Basis disease & $25(65.79)$ \\
None & $12(31.58)$ \\
Hypertension & $1(2.63)$ \\
Heart disease & $2(5.26)$ \\
Diabetes & \\
Tumour location & $3(7.89)$ \\
Proximal third & $21(55.26)$ \\
Middle third & $14(36.84)$ \\
Distal third & \\
Immunotherapeutic drugs & $21(55.26)$ \\
Pembrolizumab & $12(31.58)$ \\
Camrelizumab & $5(13.16)$ \\
Sintilimab & $5[2-13]$ \\
Interval from completion of NACl to & \\
surgery [weeks] & \\
\hline ECOG, eastern cooperative oncology group; NACl, neoadjuvant \\
chemotherapy combined with immunotherapy.
\end{tabular}

Table 2 Post NACI CT evaluation

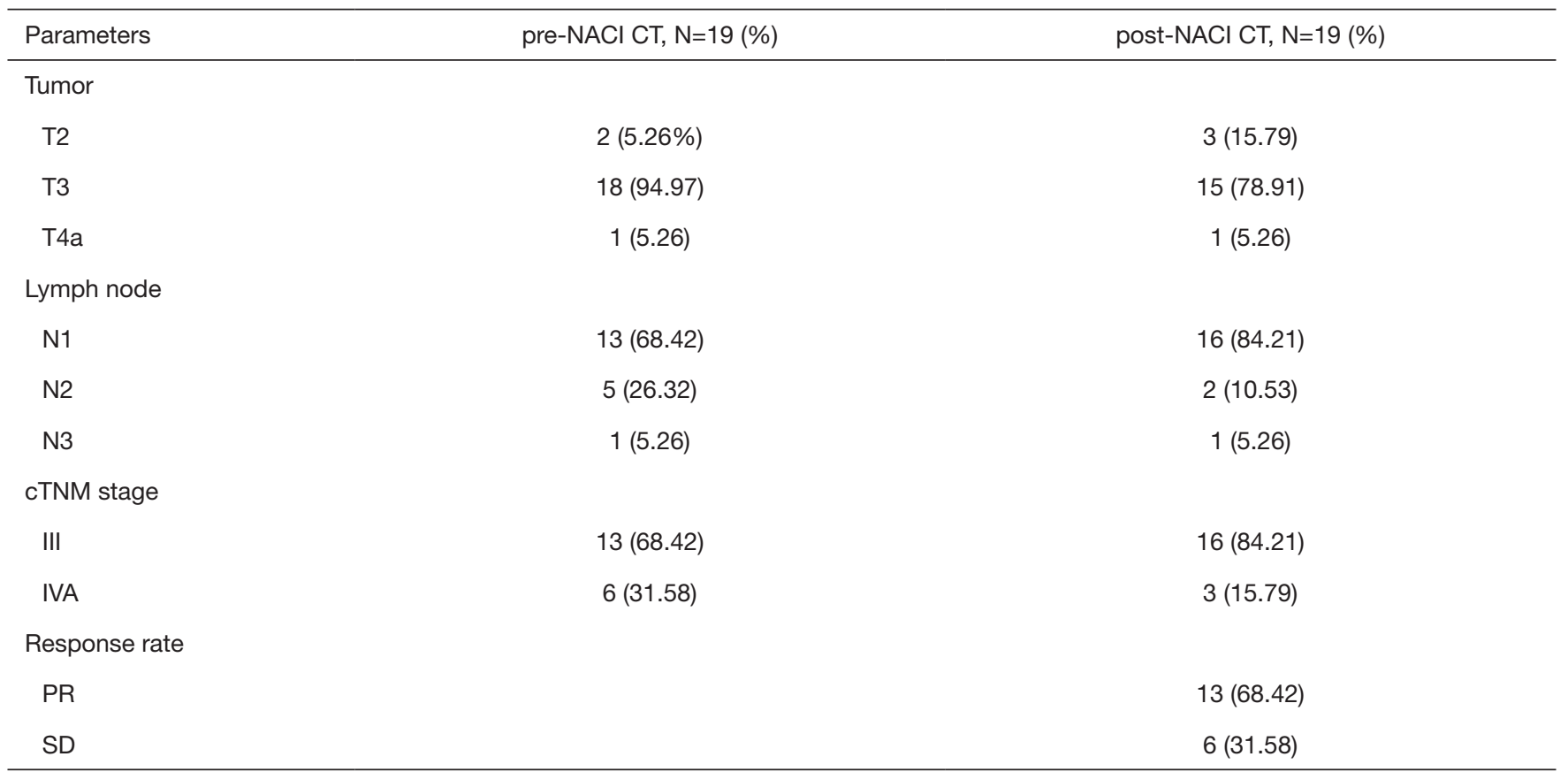

$\mathrm{NACl}$, neoadjuvant chemotherapy combined with immunotherapy. 
Table 3 The operative information and postoperative pathological information of the patients

\begin{tabular}{|c|c|}
\hline Parameters & $\mathrm{N}=38(\%)$ \\
\hline \multicolumn{2}{|l|}{ Surgery type } \\
\hline McKewon & $17(44.74)$ \\
\hline Ivor Lewis & $21(55.26)$ \\
\hline \multicolumn{2}{|l|}{ Extent of lymphadenectomy } \\
\hline Two field & 37 (97.37) \\
\hline Three field & $1(2.63)$ \\
\hline Harvested lymph nodes [No.] & $27[8-61]$ \\
\hline \multicolumn{2}{|l|}{ Extent of resection } \\
\hline Ro & $35(92.11)$ \\
\hline $\mathrm{R} 1$ & $3(7.89)$ \\
\hline Operation time (min) & $211.92 \pm 6.57$ \\
\hline Blood loss (mL) & $186.8 \pm 42.82$ \\
\hline \multicolumn{2}{|l|}{ Blood transfusion } \\
\hline Yes & $1(2.63)$ \\
\hline No & $37(97.37)$ \\
\hline \multicolumn{2}{|l|}{ Postoperative complications } \\
\hline None & $28(73.68)$ \\
\hline Pneumonia & $9(23.68)$ \\
\hline Chylothorax & $1(2.63)$ \\
\hline Wound infection & $1(2.63)$ \\
\hline Reoperation & $1(2.63)$ \\
\hline Reintubationa & $5(13.16)$ \\
\hline ICU treatment & $5(13.16)$ \\
\hline \multicolumn{2}{|l|}{ Clavien-Dindo grade } \\
\hline 0 & $28(73.68)$ \\
\hline $1-2$ & $4(10.53)$ \\
\hline $3 a$ & $1(2.63)$ \\
\hline $4 a$ & $5(13.16)$ \\
\hline Postoperative hospital stays [day] & $12[9-58]$ \\
\hline \multicolumn{2}{|l|}{ Tumor } \\
\hline T0 & $13(34.21)$ \\
\hline T1a & $2(5.26)$ \\
\hline $\mathrm{T} 1 \mathrm{~b}$ & $5(13.16)$ \\
\hline $\mathrm{T} 2$ & $5(13.16)$ \\
\hline T3 & $13(34.21)$ \\
\hline
\end{tabular}

Table 3 (continued)
Table 3 (continued)

\begin{tabular}{lc}
\hline Parameters & $\mathrm{N}=38(\%)$ \\
\hline Lymph node & $20(52.63)$ \\
N0 1 & $8(21.05)$ \\
N2 & $5(13.16)$ \\
N3 & $5(13.16)$ \\
YpTNM & \\
0 & $13(34.21)$ \\
IA & $2(5.26)$ \\
IB & $2(5.26)$ \\
IIA & $1(2.63)$ \\
IIB & $6(15.79)$ \\
IIIA & $1(2.63)$ \\
IIIB & $10(26.32)$ \\
IVA & $3(7.89)$ \\
Pathological response rate & $13(34.21)$ \\
pCR & $16(42.11)$ \\
MPR & \\
\hline pCR, complete pathological response; MPR, major pathological \\
response.
\end{tabular}

(92.11\%). A total of 10 patients $(26.32 \%)$ developed postoperative complications, including 5 patients with Clavien-Dindo grade 4a complications (13.16\%), which were transferred to the ICU for treatment. Through postoperative pathology, we found the postoperative pathology showed that 9 patients $(23.68 \%)$ were between IA-IIB stage, and 14 patients $(36.84 \%)$ were between IIIA-IVA (Table 3). A total of $13(34.21 \%)$ patients had complete pathologic response (cPR), and $16(42.11 \%)$ patients achieved MPR (Table 3). The distribution of pathologic response can be seen more visually through the waterfall plot (Figure 1).

Among 19 patients with the Continuous CT images, we found that the regression rate of SLD was significantly positively correlated with the pathological remission rate $(\mathrm{P}=0.012, \mathrm{r}=0.565)$, but the regression rate of $\mathrm{LSD}$ reduction has no correlation with pathological remission rate $(\mathrm{P}=0.262, \mathrm{r}=0.278)$. Although there was a trend between the rate of SLD regression and the rate of LSD reduction, the $\mathrm{P}$ value $(\mathrm{P}=0.096, \mathrm{r}=0.393)$ indication was not 


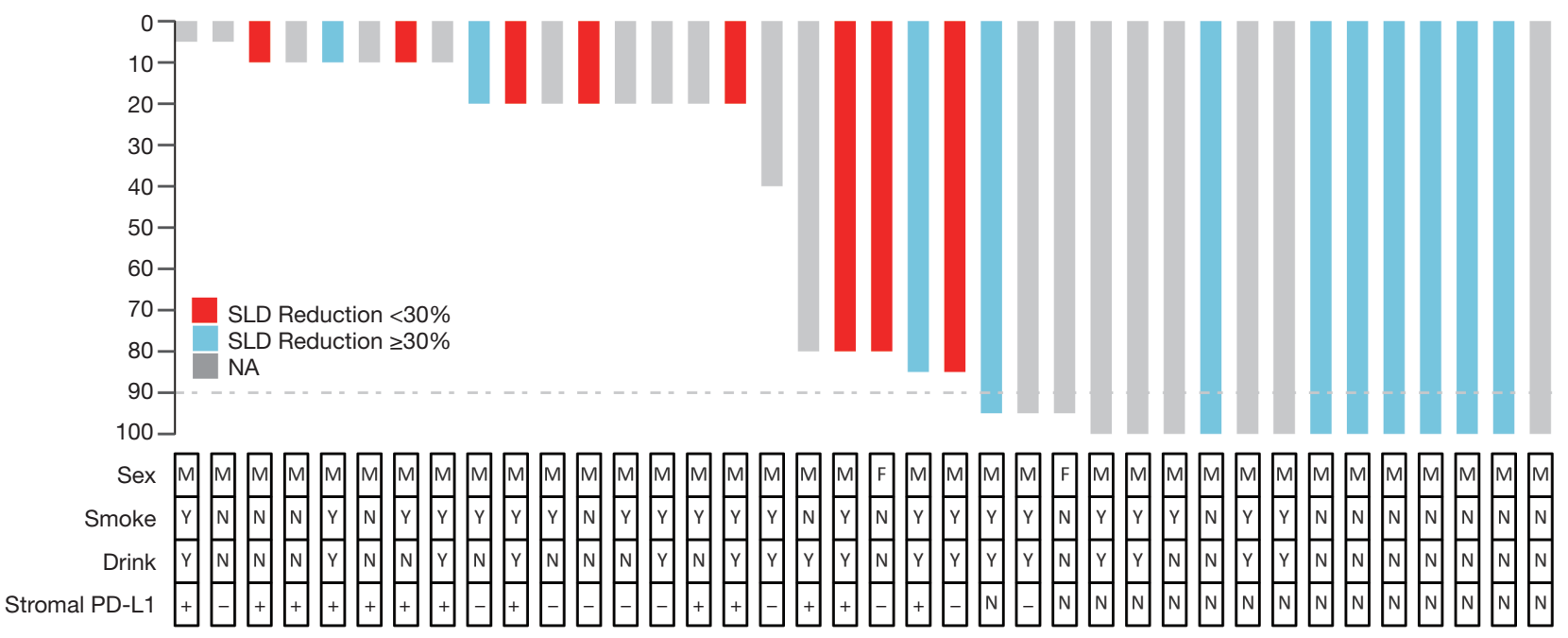

Figure 1 Waterfall plot of pathological regression.
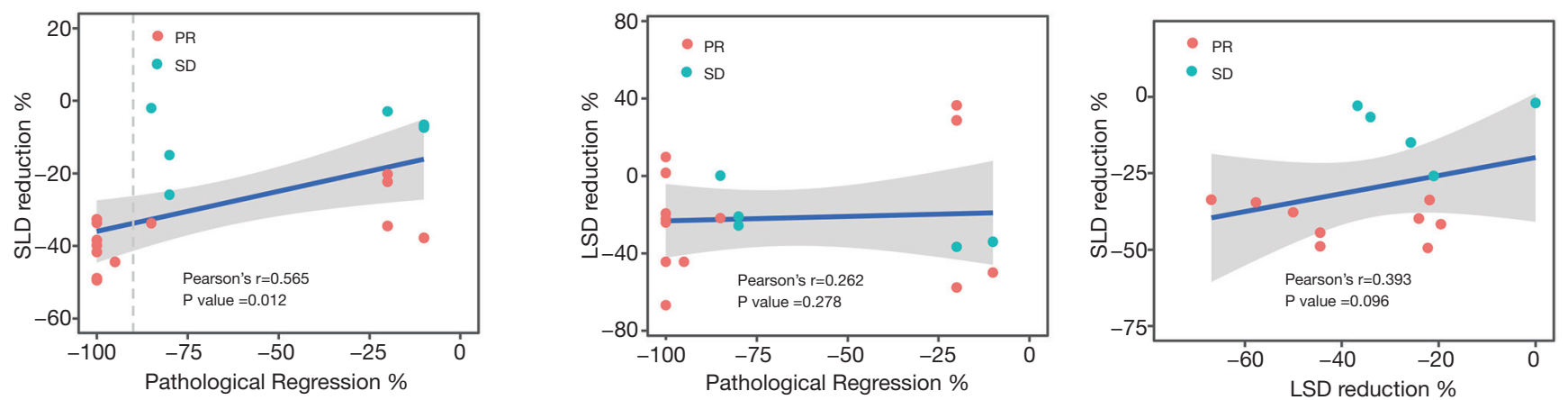

Figure 2 Correlation of pathological regression, SLD reduction, lymph node shortest diameter reduction.

meaningful (Figure 2).

In addition, we found that age $(\mathrm{P}=0.019)$ of patients were significantly different between MPR and non-MPR patients, but no significant differences were found in gender, BMI, smoking history, drinking history, tumor location, choice of drugs, clinical stage of the tumor (Table 4), HER2, P16, PD-L1 and Ki-67 (Table 5). Further, through multivariate analysis, we found none of the factors affected the MPR rate (Table 6).

\section{Discussion}

Thirty-eight patients with NACI after surgery were retrospectively analyzed, we found that not only chemotherapy combined immunotherapy has good security, and it did not increase the complications of surgery and postoperative pathological ease the situation, we learned that new adjuvant chemotherapy combined immunotherapy has higher pathological remission rate, suggested it may have a better prognosis, and, through the correlation analysis we found that the rate of SLD regression and pathological remission rate has a certain positive correlation

In recent years, immunotherapy, represented by immune checkpoint inhibitors, has become a research hotspot in treating solid tumors, and attempts and breakthroughs have also been made in esophageal cancer. For advanced esophageal cancer, including KEYNOTE-181 (10), ATTRACTION-3 (11), SHR-1210 (17), etc., studies have confirmed the potential of immunotherapy in the treatment of esophageal cancer. For locally advanced esophageal cancer, ASCO (2019) reported a pilot experiment of nivolumab combined with CRT, a total of 16 patients with 
Table 4 Correlation between preoperative basic patient information and partial pathological response

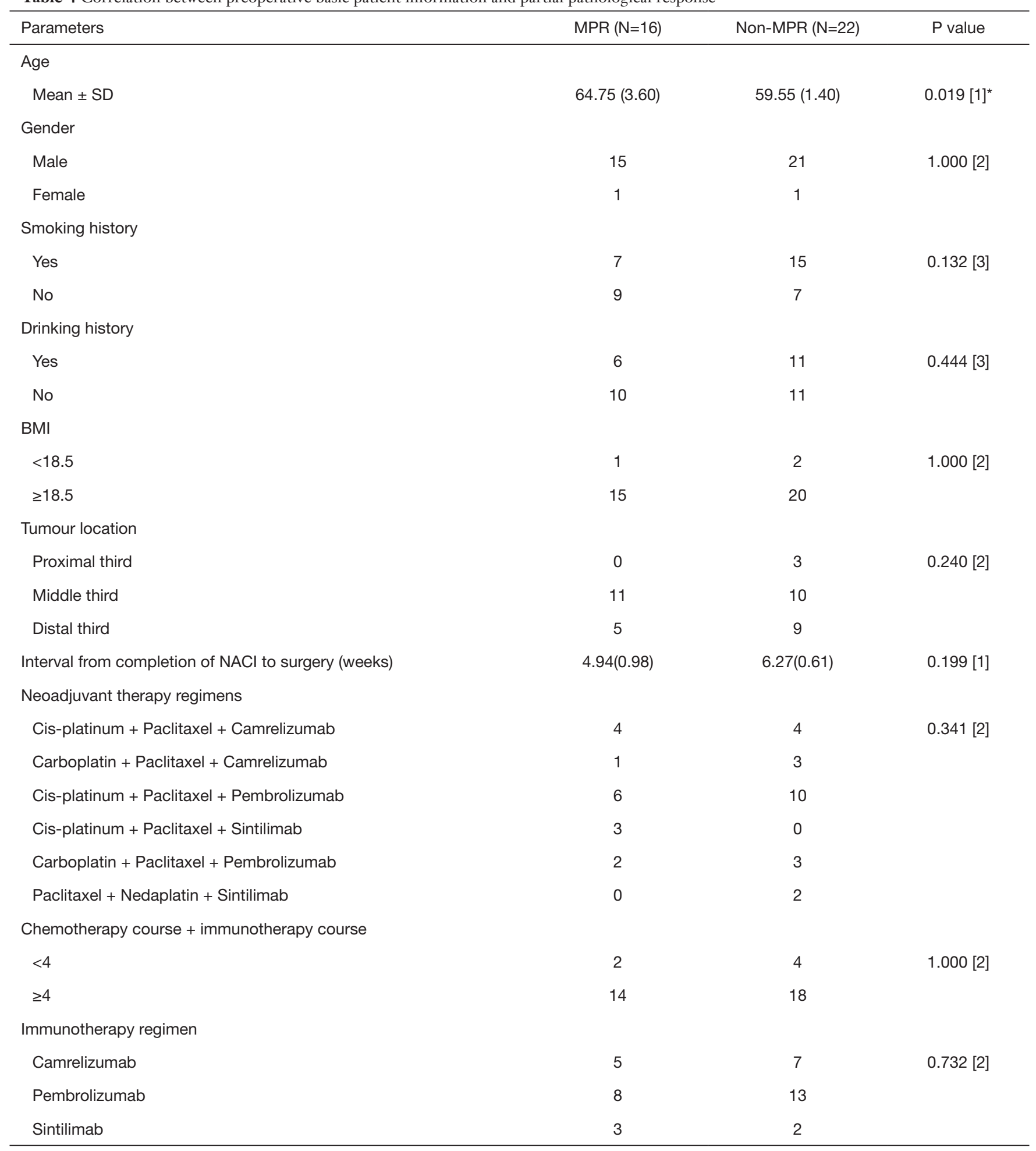

Table 4 (continued) 
Table 4 (continued)

\begin{tabular}{|c|c|c|c|}
\hline Parameters & MPR $(\mathrm{N}=16)$ & Non-MPR (N=22) & $P$ value \\
\hline$<2$ & 2 & 3 & 1.000 [2] \\
\hline$\geq 2$ & 14 & 19 & \\
\hline \multicolumn{4}{|l|}{ Staging } \\
\hline IVA & 4 & 3 & \\
\hline
\end{tabular}

[1]: $P$ value is calculated by $t$-test; [2]: $P$ value is calculated by Fisher exact test; [3]: $P$ value is calculated by chi-squared test. Main Pathological Reaction, MPR; $\mathrm{P}>0.05 \mathrm{NS},{ }^{*} \mathrm{P}<0.05$.

Table 5 Correlation between Pathological immunohistochemistry information and partial pathological response

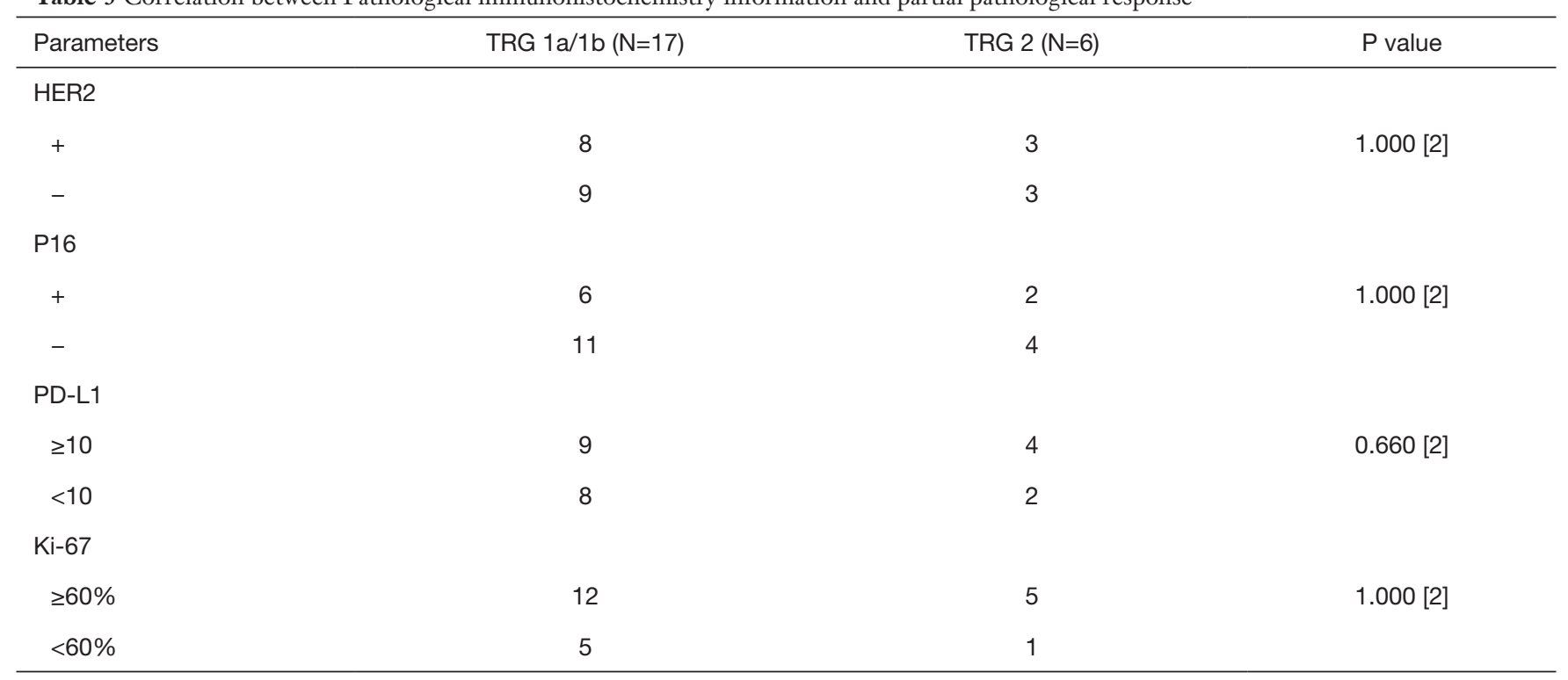

[2]: $P$ value is calculated by Fisher exact test; $P>0.05$ NS.

Table 6 Multivariate analysis of the relationship between patient's basic information and the effect of immunotherapy

\begin{tabular}{lccc}
\hline Variables & OR & 95\% Cl & 0.101 \\
\hline Age & 1.15 & $0.97-1.35$ & 0.223 \\
Smoking history & 7.23 & $0.3-173.78$ & 0.669 \\
Drinking history & 0.56 & $0.04-8.21$ & 0.359 \\
Tumour location & 0.44 & $0.08-2.51$ & 0.089 \\
Interval from completion of NACl to surgery (weeks) & 0.93 & $0.86-1.01$ & 0.118 \\
Immunotherapy regimen & 4.35 & $0.69-27.61$ & 0.599 \\
NACl course & 0.78 & $0.31-1.97$ & 0.194 \\
Clinical stage & 0.19 & $0.01-2.34$ & \\
\hline
\end{tabular}

$P$ value is calculated by Binary logistic regression curve. All variables were included. 
stage II-III esophageal cancer or gastroesophageal junction cancer were enrolled. Five patients achieved complete pathological remission, 9 patients achieved pathological downgrade, and 15 patients achieved $\mathrm{R} 0$ resection (18). The ASCO (2019) meeting also reported the results of Avelumab combined with neoadjuvant chemotherapy and radiotherapy in the treatment of operable locally advanced esophageal adenocarcinoma. Also, it achieved gratifying results, which is better than the results of previous CROSS studies $(19,20)$.

By comparing our study to our previous trial, the operation time $(211.92 \pm 6.57$ vs. $174-202 \mathrm{~min})$, intraoperative blood loss $(186.8 \pm 42.82$ vs. $103-325 \mathrm{~mL})$, and postoperative complication (26.32\% vs. $30-41 \%)$ were indicating that the quality of our neoadjuvant treatment did not increase the risk of surgery, and it has good safety $(21,22)$.

Patients who get MPR after neoadjuvant therapy are more likely to have better survival $(23,24)$. The pathological response rate of the two chemotherapy drugs in the past was around $2.5-33 \%(25,26)$, and the KEYNOTE-181 (10) experiment showed that postoperative pathology of 18 evaluable patients found MPR in $9(50 \%)$ cases and pathological complete remission (pCR) in 3 cases (17\%). Recently, KEYNOTE-590 (27) is the world's first phase III clinical study for the first-line immune treatment of locally advanced or metastatic esophageal cancer, enrolled a total of 749 patients. The results showed that the objective response rate (ORR) of patients in the first-line treatment group of pembrolizumab combined with chemotherapy was $45 \%$, which was significantly higher than that of the chemotherapy group $(29.3 \%)(\mathrm{P}<0.0001)$. Our results showed that after NACI, the complete response rate and PR rate of esophageal cancer patients were $34.21 \%$ and $42.11 \%$, respectively. The pCR rate was significantly higher ( $34 \%$ vs. $3.8 \%$ ) compare with the patients treated with chemotherapy (28).

In past reports, we know that in the ATTRACTION-3 experiment, nivolumab is the survival benefit of patients with esophageal cancer has nothing to do with tumor PDL1 expression and PD-L1 negative ESCC patients also respond to pembrolizumab (11). In the single-arm Phase II KEYNOTE-180 study, the ORR of patients with PDL1 negative tumors was $6.3 \%$, while that of patients with PD-L1 positive tumors was $13.8 \%$ (29). Low remission rates combined with sample size limitations, the difference of PD-L1 in our study has not been shown. In this study, however, in comparing MPR and non-MPR, except for the significant difference in age, no significant difference was found in other factors, including gender, smoking history, Immunotherapy regimen, PD-L1, and Ki-67, etc. Further, we conducted a multi-factor analysis and found that the difference in age is affected by other factors, which has no decisive influence on the judgment of MPR, and there was still no significant difference in efficacy between the different immunotherapy regimens. In the future, we need to look for indicators to predict MPR.

In many cancers, scientists always hope to find immunotherapy indicators that can effectively indicate the effect of immunotherapy, which can help the choice of subsequent treatment options. In this study, there is a positive correlation between the patient's SLD reduction rate and the pathological remission rate $(r=0.565, \mathrm{P}=0.012)$, but no apparent correlation between the changes of LSD reduction rate and SLD reduction rate $(\mathrm{r}=0.393, \mathrm{P}=0.096)$ and the pathological remission rate $(\mathrm{r}=0.262, \mathrm{P}=0.278)$. In the past reports, the patient's SLD reduction rate and the pathological remission rate have always been related to the trend, including lung cancer (30), etc. This may be because the esophagus, as an organ of the digestive tract, has strong peristalsis and contraction movement, and due to the scraping of the food during the eating process, the necrotic cancer tissue is peeled off into the stomach so that its changes can be observed on imaging.

There are some limitations to our study. Firstly, the paper's sample size is small and retrospective; secondly, some patients have disease progression and cannot be operated on, so there may be a deviation in patient selection. However, this paper has confirmed the safety of surgery after immunotherapy, and it is the first retrospective analysis to compare the safety of different immunodrugs. Further research is needed to clarify the value of neoadjuvant immunotherapy.

\section{Conclusions}

The rate of MPR in ESCC patients reached $42.11 \%$. The use of the NACI regimen may not increase the occurrence of complications in neoadjuvant treatment and operation, and the SLD regression rate has a certain guiding significance for the effect of immunotherapy.

\section{Acknowledgments}

We thank the patient to provide the samples for this study. We also thank Xu Pan from XMU School of Pharmaceutical Sciences-Amogene Joint R\&D Center 
for Genetic Diagnostics, Amogene Biotech for excellent technical assistance.

Funding: This work was supported by Ministry of Science and Technology of the People's Republic of China (2017YFA0505500; 2016YFA0501800), Science and Technology Commission of Shanghai Municipality (19XD1401300).

\section{Footnote}

Reporting Checklist: The authors have completed the STROBE reporting checklist. Available at http://dx.doi. org/10.21037/jtd-21-340

Data Sharing Statement: Available at http://dx.doi. org/10.21037/jtd-21-340

Conflicts of Interest: All authors have completed the ICMJE uniform disclosure form (available at http://dx.doi. org/10.21037/jtd-21-340). The authors have no conflicts of interest to declare.

Ethical Statement: The authors are accountable for all aspects of the work in ensuring that questions related to the accuracy or integrity of any part of the work are appropriately investigated and resolved. The study was conducted in accordance with the Declaration of Helsinki (as revised in 2013). The study was approved by the Fudan University Shanghai Cancer Center Institutional Review Board (No. 090977-1) and written informed consent was obtained from all patients.

Open Access Statement: This is an Open Access article distributed in accordance with the Creative Commons Attribution-NonCommercial-NoDerivs 4.0 International License (CC BY-NC-ND 4.0), which permits the noncommercial replication and distribution of the article with the strict proviso that no changes or edits are made and the original work is properly cited (including links to both the formal publication through the relevant DOI and the license). See: https://creativecommons.org/licenses/by-nc-nd/4.0/.

\section{References}

1. Bray F, Ferlay J, Soerjomataram I, et al. Global cancer statistics 2018: GLOBOCAN estimates of incidence and mortality worldwide for 36 cancers in 185 countries. CA Cancer J Clin 2018;68:394-424.
2. Rice TW, Ishwaran H, Ferguson MK, et al. Cancer of the Esophagus and Esophagogastric Junction: An Eighth Edition Staging Primer. J Thorac Oncol 2017;12:36-42.

3. Kelsen DP, Ginsberg R, Pajak TF, et al. Chemotherapy followed by surgery compared with surgery alone for localized esophageal cancer. N Engl J Med 1998;339:1979-84.

4. Kelsen DP, Winter KA, Gunderson LL, et al. Longterm results of RTOG trial 8911 (USA Intergroup 113): a random assignment trial comparison of chemotherapy followed by surgery compared with surgery alone for esophageal cancer. J Clin Oncol 2007;25:3719-25.

5. Cunningham D, Allum WH, Stenning SP, et al. Perioperative chemotherapy versus surgery alone for resectable gastroesophageal cancer. $\mathrm{N}$ Engl J Med 2006;355:11-20.

6. van Hagen P, Hulshof MC, van Lanschot JJ, et al. Preoperative chemoradiotherapy for esophageal or junctional cancer. N Engl J Med 2012;366:2074-84.

7. Kelly RJ, Ajani JA, Kuzdzal J, et al. Adjuvant Nivolumab in Resected Esophageal or Gastroesophageal Junction Cancer. N Engl J Med 2021;384:1191-203.

8. Zhang W, Wang P, Pang Q. Immune checkpoint inhibitors for esophageal squamous cell carcinoma: a narrative review. Ann Transl Med 2020;8:1193.

9. Boonstra JJ, Kok TC, Wijnhoven BP, et al. Chemotherapy followed by surgery versus surgery alone in patients with resectable oesophageal squamous cell carcinoma: longterm results of a randomized controlled trial. BMC Cancer 2011;11:181.

10. Kojima T, Shah MA, Muro K, et al. Randomized Phase III KEYNOTE-181 Study of Pembrolizumab Versus Chemotherapy in Advanced Esophageal Cancer. J Clin Oncol 2020;38:4138-48.

11. Kato K, Cho BC, Takahashi M, et al. Nivolumab versus chemotherapy in patients with advanced oesophageal squamous cell carcinoma refractory or intolerant to previous chemotherapy (ATTRACTION-3): a multicentre, randomised, open-label, phase 3 trial. Lancet Oncol 2019;20:1506-17.

12. Li B, Chen H, Xiang J, et al. Pattern of lymphatic spread in thoracic esophageal squamous cell carcinoma: A single-institution experience. J Thorac Cardiovasc Surg 2012;144:778-85; discussion 785-6.

13. Nishimaki T, Tanaka O, Ando N, et al. Evaluation of the accuracy of preoperative staging in thoracic esophageal cancer. Ann Thorac Surg 1999;68:2059-64.

14. Yip HC, Shirakawa Y, Cheng CY, et al. Recent advances in minimally invasive esophagectomy for squamous 
esophageal cancer. Ann N Y Acad Sci 2020;1482:113-20.

15. Eisenhauer EA, Therasse P, Bogaerts J, et al. New response evaluation criteria in solid tumours: revised RECIST guideline (version 1.1). Eur J Cancer 2009;45:228-47.

16. Japan Esophageal S. Japanese Classification of Esophageal Cancer, 11th Edition: part I. Esophagus 2017;14:1-36.

17. Zhang B, Wang X, Li Q, et al. Efficacy of irinotecan-based chemotherapy after exposure to an anti-PD-1 antibody in patients with advanced esophageal squamous cell carcinoma. Chin J Cancer Res 2019;31:910-7.

18. Kelly RJ, Smith KN, Anagnostou V, et al. Neoadjuvant nivolumab plus concurrent chemoradiation in stage ii/iii esophageal/gastroesophageal junction cancer. J Clin Oncol 2019;37:142.

19. Uboha NV, Maloney JD, Mccarthy D, et al. Safety of neoadjuvant chemoradiation (CRT) in combination with avelumab (A) in the treatment of resectable esophageal and gastroesophageal junction (E/GEJ) cancer. J Clin Oncol 2019;37:abstr 4041.

20. Shapiro J, van Lanschot JJB, Hulshof M, et al. Neoadjuvant chemoradiotherapy plus surgery versus surgery alone for oesophageal or junctional cancer (CROSS): long-term results of a randomised controlled trial. Lancet Oncol 2015;16:1090-8.

21. Li B, Zhang Y, Miao L, et al. Esophagectomy With ThreeField Versus Two-Field Lymphadenectomy for Middle and Lower Thoracic Esophageal Cancer: Long-Term Outcomes of a Randomized Clinical Trial. J Thorac Oncol 2021;16:310-7.

22. Li B, Xiang J, Zhang Y, et al. Comparison of IvorLewis vs Sweet esophagectomy for esophageal squamous cell carcinoma: a randomized clinical trial. JAMA Surg 2015;150:292-8

23. Heneghan HM, Donohoe C, Elliot J, et al. Can CTPET and Endoscopic Assessment Post-Neoadjuvant

Cite this article as: $\mathrm{Wu} Z$, Zheng Q, Chen $\mathrm{H}$, Xiang J, Hu H, Li H, Pan Y, Peng Y, Yao X, Liu P, Sun Y, Li B, Zhang Y. Efficacy and safety of neoadjuvant chemotherapy and immunotherapy in locally resectable advanced esophageal squamous cell carcinoma. J Thorac Dis 2021;13(6):3518-3528. doi: $10.21037 /$ jtd-21-340
Chemoradiotherapy Predict Residual Disease in

Esophageal Cancer? Ann Surg 2016;264:831-8.

24. Rice TW, Lerut TE, Orringer MB, et al. Worldwide Esophageal Cancer Collaboration: neoadjuvant pathologic staging data. Dis Esophagus 2016;29:715-23.

25. Hayata K, Ojima T, Nakamori M, et al. Neoadjuvant Chemotherapy with Docetaxel, Cisplatin and S-1 for Resectable Advanced Esophageal Cancer. Anticancer Res 2018;38:5267-73.

26. Ando N, Kato H, Igaki H, et al. A randomized trial comparing postoperative adjuvant chemotherapy with cisplatin and 5-fluorouracil versus preoperative chemotherapy for localized advanced squamous cell carcinoma of the thoracic esophagus (JCOG9907). Ann Surg Oncol 2012;19:68-74.

27. Kato K, Shah MA, Enzinger P, et al. KEYNOTE-590: Phase III study of first-line chemotherapy with or without pembrolizumab for advanced esophageal cancer. Future Oncol 2019;15:1057-66.

28. Wang H, Tang H, Fang Y, et al. Morbidity and Mortality of Patients Who Underwent Minimally Invasive Esophagectomy After Neoadjuvant Chemoradiotherapy vs Neoadjuvant Chemotherapy for Locally Advanced Esophageal Squamous Cell Carcinoma: A Randomized Clinical Trial. JAMA Surg 2021;156:444-51.

29. Shah MA, Kojima T, Hochhauser D, et al. Efficacy and Safety of Pembrolizumab for Heavily Pretreated Patients With Advanced, Metastatic Adenocarcinoma or Squamous Cell Carcinoma of the Esophagus: The Phase 2 KEYNOTE-180 Study. JAMA Oncol 2019;5:546-50.

30. Li N, Ying J, Tao X, et al. Efficacy and safety of neoadjuvant PD-1 blockade with sintilimab in resectable squamous non-small cell lung cancer (sqNSCLC). J Clin Oncol 2019;37:abstr 8531. 